\title{
A Biometrical Genome Search in Rats Reveals the Multigenic Basis of Blood Pressure Variation
}

\author{
Nicholas J. Schork, ${ }^{1,2,8}$ Josè E. Krieger, ${ }^{3,4}$ Maria R. Trolliet, ${ }^{5}$ \\ Klebber G. Franchini, ${ }^{3}$ George Koike, ${ }^{4,5}$ Eduardo M. Krieger, ${ }^{3}$ \\ Eric S. Lander, ${ }^{6,7}$ Victor J. Dzau, ${ }^{4}$ and Howard J. Jacob ${ }^{5}$
}

${ }^{1}$ Department of Genetics, Case Western Reserve University, Cleveland, Ohio 44106-4955; ${ }^{2}$ The Jackson Laboratory, Bar Harbor, Maine 04609; ${ }^{3}$ Sao Paulo Heart Institute, University of Sao Paulo, Sao Paulo, Brazil; ${ }^{4}$ Cardiovascular Research Center, Stanford University, Palo Alto, California 94305-1901; ${ }^{5}$ Cardiovascular Research Center, Massachusetts General Hospital, Charlestown, Massachusetts 02137; ${ }^{6}$ Whitehead Institute for Biomedical Research, Cambridge, Massachusetts 02142; ${ }^{7}$ Department of Biology, Massachussetts Institute of Technology, Cambridge, Massachusetts 02139

A genome-wide search for multiple loci influencing salt-loaded systolic blood pressure (NaSBP) variation among $188 \mathrm{~F}_{2}$ progeny from a cross between the Brown-Norway and spontaneously hypertensive rat strains was pursued in an effort to gain insight into the polygenic basis of blood pressure regulation. The results suggest that loci within five to six genomic regions collectively explain $\sim 43 \%$ of the total NaSBP variation exhibited among the $188 \mathrm{~F}_{2}$ progeny. Many of these loci are in regions that previous studies have not implicated in blood pressure regulation. Ultimately, however, this study not only sheds light on the multigenic basis of blood pressure but provides further evidence that the identification of the genetic determinants of polygenic traits in mammals is possible with modern biometrical and molecular genetic tools in controlled settings (i.e., breeding paradigm and model organism).

Modern methods for manipulating and isolating segments of DNA have allowed researchers to examine the genetic basis of traits exhibited by a wide variety of organisms (Lander and Schork 1994). However, the vast majority of traits whose genetic bases have been examined to date with these methods either exhibit largely monogenic and overtly Mendelian patterns of inheritance, or are associated with plants (Tanksley 1993; Lander and Schork 1994). Exceptions include recent studies on the genetics of type I diabetes in mice and humans and fatness in pigs (Risch et al. 1993; Andersson et al. 1994; Davies et al. 1994; Hashimoto et al. 1994). One set of traits whose genetic bases have been particularly difficult to disclose are quantitative traits in mammals. Quantitative traits show continuous variation in the population at large (e.g., height and cholesterol level)

${ }^{8}$ Corresponding author. Some of the work described in this paper was conducted at the Division of Hypertension, Department of Medicine, and Department of Epidemiology, University of Michigan, Ann Arbor, Michigan 48109.

E-MAIL njs2@po.cwru.edu; FAX (216) 368-5857. and are difficult to study from genetic standpoints in many organisms because they are often influenced by a number of genes and environmental factors. This characteristic polygenic or "multifactorial" basis of quantitative traits makes it difficult to isolate and characterize each and every factor influencing them, because any one factor may have only a (relatively) slight effect on a relevant trait and thereby obscure its ultimate contribution to the trait (Tanksley 1993; Lander and Schork 1994).

Blood pressure (BP) is a prototypical quantitative trait known to have numerous biochemical, physiological, environmental, and genetic determinants (Ward 1990). Although recent studies investigating BP in inbred rats have isolated genes influencing BP regulation, these studies have ultimately determined, or focused on, one or two loci that individually explain only a small fraction of the total BP variation exhibited by the populations studied, and as such did not make explicit attempts in their analyses to accommodate the probable collective effects of 
multiple loci on BP regulation (Pravenec et al. 1989, 1991; Rapp et al. 1989, 1994; Hilbert et al. 1991; Jacob et al. 1991; Hamet et al. 1992; Dubay et al. 1993; Harris et al. 1993; Lindpaintner et al. 1993; Samani et al. 1993; Deng et al. 1994a,b). We report here the results of a study designed to simultaneously assess multiple loci influencing systolic blood pressure in rats after a salt challenge (NaSBP). A suite of traditional and novel biometrical and molecular genetic techniques were used to carry out the study. The results suggest that five to six loci collectively explain between $31 \%$ and $43 \%$ of the total NaSBP variation exhibited by the rats studied. These results shed light on the multigenic basis of blood pressure and provide further evidence that the genetic determinants of multigenic traits in mammals may yield to modern biometrical "genome-scan" technologies in controlled settings.

\section{RESULTS}

Table 1 describes 17 marker loci that met the $P$-value criteria out of the total of 179 markers tested during stage 1 of our analysis. Mean geno- type effects, test statistic $P$-values for the analysis of variance (ANOVAs), and the best-fitting genetic models for each locus are given. Two things should be noted from Table 1 . First, many of the 17 markers are on the same chromosome, suggesting that some of them have produced significant results merely because they are in possible linkage disequilibrium with a quantitative trait locus (QTL) linked to another marker that is significant. Second, the mean salt-loaded systolic blood pressure (NaSBP) values are generally consistent with the best-fitting genetic regression model, as expected. Thus, for example, the best fitting genetic regression model associated with marker R762 was recessive, and, as can be seen from the mean values recorded for the genotypes at that locus, rats either homozygous for the $\mathrm{BN}$ allele or heterozygous have similar mean NaSBP values, whereas rats homozygous for the SHR allele have a significantly higher NaSBP mean value.

The 17 marker loci described in Table 1 were entered into a stepwise multiple regression analysis as described in Methods. The final model determined from the stepwise regression procedure

Table 1. Mean (S.E.) and ANOVA results for markers passing univariate criteria (see text) for consideration as residing near or on putative QTLs

\begin{tabular}{lclllllll}
\hline Marker & Chr & $n$ & B/B & B/S & S/S & ANOV $\boldsymbol{P}$ & K-W $\boldsymbol{P}$ & Mode \\
\hline IGF2 & 1 & 163 & $139.61 \pm 2.20$ & $149.70 \pm 1.58$ & $147.61 \pm 1.35$ & 0.0041 & 0.0038 & $\mathrm{D}$ \\
R360 & 2 & 169 & $143.32 \pm 2.03$ & $146.82 \pm 1.41$ & $155.09 \pm 3.14$ & 0.0018 & 0.0032 & $\mathrm{C}$ \\
P9K & 2 & 174 & $142.21 \pm 2.10$ & $147.23 \pm 1.60$ & $155.90 \pm 3.11$ & 0.0009 & 0.0017 & $\mathrm{C}$ \\
PKLG & 2 & 171 & $142.57 \pm 2.10$ & $146.34 \pm 1.55$ & $153.91 \pm 2.67$ & 0.0036 & 0.0041 & $\mathrm{C}$ \\
GCA & 2 & 167 & $142.20 \pm 2.15$ & $146.19 \pm 1.56$ & $153.13 \pm 2.77$ & 0.0078 & 0.0077 & $\mathrm{C}$ \\
R5159 & 2 & 163 & $143.58 \pm 2.29$ & $146.81 \pm 1.61$ & $156.94 \pm 3.64$ & 0.0027 & 0.0072 & $\mathrm{R}$ \\
R480 & 2 & 153 & $141.31 \pm 2.33$ & $146.82 \pm 1.41$ & $152.65 \pm 3.02$ & 0.0048 & 0.0019 & $\mathrm{C}$ \\
R155 & 2 & 169 & $142.15 \pm 2.00$ & $147.23 \pm 1.60$ & $153.62 \pm 3.04$ & 0.0039 & 0.0030 & $\mathrm{D}$ \\
MT1PB & 2 & 172 & $141.29 \pm 1.97$ & $146.82 \pm 1.54$ & $156.22 \pm 2.86$ & 0.0001 & 0.0002 & $\mathrm{C}$ \\
R5129 & 2 & 170 & $141.21 \pm 2.10$ & $147.95 \pm 1.52$ & $155.59 \pm 3.51$ & 0.0005 & 0.0016 & $\mathrm{C}$ \\
AMPL & 4 & 169 & $143.11 \pm 2.26$ & $145.84 \pm 1.62$ & $155.01 \pm 2.90$ & 0.0020 & 0.0040 & $\mathrm{R}$ \\
R995 & 4 & 167 & $142.68 \pm 2.32$ & $145.90 \pm 1.61$ & $153.53 \pm 2.56$ & 0.0058 & 0.0057 & $\mathrm{C}$ \\
SPRD & 4 & 168 & $141.07 \pm 2.40$ & $146.74 \pm 1.62$ & $154.44 \pm 2.71$ & 0.0010 & 0.0005 & $\mathrm{C}$ \\
NPY2 & 4 & 163 & $140.74 \pm 2.14$ & $147.31 \pm 1.71$ & $151.04 \pm 2.21$ & 0.0097 & 0.0060 & $\mathrm{C}$ \\
R514 & 4 & 168 & $140.52 \pm 2.52$ & $147.63 \pm 1.87$ & $152.91 \pm 2.01$ & 0.0038 & 0.0007 & $\mathrm{C}$ \\
R19 & 8 & 170 & $142.47 \pm 2.04$ & $145.74 \pm 1.66$ & $152.75 \pm 2.38$ & 0.0050 & 0.0077 & $\mathrm{C}$ \\
R762 & 16 & 169 & $144.40 \pm 2.40$ & $145.00 \pm 1.56$ & $155.62 \pm 2.84$ & 0.0007 & 0.0027 & $\mathrm{R}$ \\
\hline
\end{tabular}

(Marker) Marker locus name; (Chr) chromosomal location of the marker; $(n)$ sample size (i.e., number of organisms typed for the marker locus; (B/B) BN/BN genotype homozygote mean NaSBP and S.E for the marker locus; $(B / S)$ heterozygote mean NaSBP and S.E.; (S/S) SHR/SHR genotype homozygote NaSBP mean and S.E.; (ANOV $P$ ) $P$ value obtained from an ANOVA test of the equality of the marker genotype means; (K-W P) $P$ value from a Kruskall-Wallis nonparametric test of the equality of the marker genotype means; (Mode) mode of inheritance [(R) recessive; (D) dominant; (C) codominant or additive] obtained from the best-fitting regression model for the marker (or associated QTL) allelic effects on NaSBP (see text). 


\section{SCHORK ET AL.}

is described in Table 2, with the markers listed in the order of their overall contribution to NaSBP variation. As can be determined from Table 2, 5 of the 17 marker loci assessed in the analysis entered the final model. These five marker loci explain $-31 \%$ of the total NaSBP variation exhibited by the rats. Note that for the codominant loci in the model, one-half times the regression coefficient reported in Table 2 represents the effect of substituting a spontaneously hypersensitive rat strain (SHR) allele for a Brown-Norway (BN) allele on NaSBP value. For dominant or recessive loci, the regression coefficient measures the increase in NaSBP attributable to NaSBPincreasing genotypes. Also note that whereas loci on chromosomes $2,4,8$, and 16 entered the final model, the IGF2 locus on chromosome 1 did not (cf. Tables 1 and 2), suggesting that either its effects as described in Table 1 were a chance occurrence or its effects are relatively weak when considered simultaneously with other locus effects. This absence of IGF2 locus effects from the final regression model illustrates the limitations of examining a single locus effect on a multifactorial trait, and emphasizes the need for multivariate models as a safeguard against false-positive univariate analytic model results. However, we emphasize that our primary phenotype is NaSBP and not basal SBP. It is possible that the IGF2 locus does influence basal (or another kind of) BP but does not necessarily impact NaSBP (or at least not to the same degree as other loci). As a further check on the impact of these five marker loci on NaSBP variation, the indicator-variable information assigned to each rat on the basis of genotype information and the best-fitting genetic regression model for each locus were summed, producing a score that reflects the number of NaSBPincreasing (i.e., likely SHR effect) alleles or genotypes possessed by each rat. As an example of the summation process, consider that an organism homozygous for the SHR allele at the R514 locus would be assigned a value of 1.0. If this organism was also heterozygous at both the R5129 and R5159 locus it would be assigned values of $1 / 2$ and 0 for these loci, respectively. Thus, this rat would be assigned a score of $1.0+0.5+0.0=1.5$ for these three loci. Such sums were made over all five loci for each rat. Figure 1 plots this number against mean NaSBP values. There is an obvious relationship between the five loci and NaSBP values, with a possible saturation or threshold in the effect of many deleterious genotypes present. The combined effect of these loci on NaSBP is nonetheless clear, as the range in NaSBP values between those possessing few and those possessing many of the five NaSBP-influencing genotypes is some $50 \mathrm{mmHg}$. Examination of possible interaction or epistatic effects involving the five loci described in Table 2 suggested that no significant interactions exist among them.

Composite interval mapping using the five marker loci entering into the final stepwise regression model as background loci was then pursued. Refinements of the locations and effects of the loci linked to the markers in the background set were also pursued, as described in Methods. Using this strategy, we were able to identify another putative QTL on chromosome 3 that did not appear to be associated with a marker whose effects were detected in the initial analysis stage. It is noteworthy that this additional QTL had alleles whose effects were opposite to the other putative QTL alleles; that is, the SHR allele at this locus was associated with lower NaSBP than the $\mathrm{BN}$ allele. A reexamination of the markers near this locus confirmed this to 


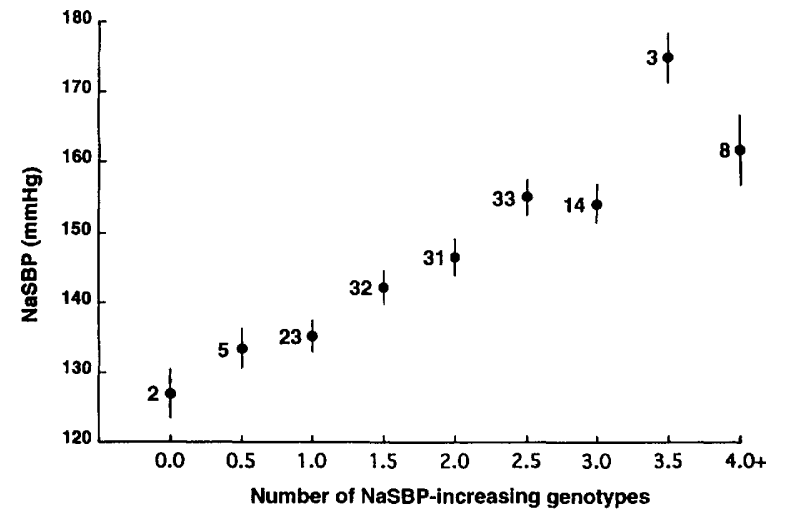

Figure 1 Number of genotypes producing increases in NaSBP possessed by the $F_{2}$ rat progeny plotted against the mean NaSBP value based on the five loci described in Table 2. (S.E.) Vertical lines. The numbers represent the number of rats with a particular number of putative NaSBP-increasing genotypes. An organism was assigned half of a NaSBPincreasing genotype if it was heterozygous at a locus showing codominance (i.e., additivity; see text).

some degree, as the genotypes at these loci showed a downward trend in NaSBP with the SHR allele. Because the BN strain is a normotensive strain as opposed to a hypotensive strain (i.e., it was bred for normal blood pressure and not low blood pressure), it is quite possible that NaSBP-increasing alleles have been fixed within the BN strain.

Expected additive and dominance effects as-
MULTIGENIC BASIS OF BLOOD PRESSURE VARIATION

sociated with the QTLs detected in the composite interval mapping analysis were computed and assessed in a final regression model. Table 3 describes the overall results of this analysis. Table 3 suggests that the six detected QTLs explain $\sim 43 \%$ of NaSBP variation in the $F_{2}$ progeny studied. If the QTLs with nonsignificant semicomposite (SC) interval mapping $F$ statistics were excluded from this model, the remaining four QTLs explain $31 \%$ of NaSBP variation. This suggests that although the preliminary evidence favoring QTL status for these two loci was weakened somewhat during the SC interval mapping modeling, these loci are important NaSBP determinants nonetheless. Exclusion of dominance effects from the model, which appear slight from Table 3, yields a model that explains $38 \%$ of NaSBP variation. This suggests that the evidence for dominance effects observed during the first stage of our analysis (e.g., Table 1) was likely caused by either our use of marker genotype information, or the univariate nature of the analysis done at that stage. Interaction terms involving the QTLs outlined in Table 3 were tested in the model also. The results suggested that these terms did not make significant contributions in the model (data not shown). Thus, we could find no evidence for overt epistatic interactions among the QTLs detected with our procedures. It is important to emphasize, however, that our assessment of interactions may have been too naive or unsophisti-

Table 3. Final interval mapping-determined QTL regression modeling results and approximate positions of the QTLs in relation to their flanking markers

\begin{tabular}{|c|c|c|c|c|c|c|c|c|c|c|}
\hline Chr & $M_{1}$ & $\mathrm{M}_{2}$ & Dist & QTL-Dist & $F$ stat & Lod & Add $R^{2}$ & Dom $R^{2}$ & Cum $\mathbf{R}^{2}$ & Effect \\
\hline 2 & MT1PB & R5129 & 4.1 & 4.1 & $5.21^{\mathrm{a}}$ & 3.0 & 8.07 & 0.03 & 8.10 & + \\
\hline 2 & GCA & R5159 & 7.8 & 2.8 & 9.14 & 6.3 & 6.03 & 1.15 & 15.28 & + \\
\hline 2 & R5229 & R598 & 23.0 & 0.0 & 7.94 & 3.0 & 4.01 & 1.47 & 20.76 & - \\
\hline 4 & NPY2 & R514 & 15.8 & 11.4 & 11.04 & 4.6 & 12.32 & 0.44 & 33.52 & + \\
\hline 8 & R19 & $\mathrm{R} 850$ & 13.9 & 6.1 & $5.93^{\mathrm{a}}$ & 5.1 & 6.77 & 0.17 & 40.46 & + \\
\hline 16 & R762 & R220 & 14.1 & 6.2 & 9.48 & 4.3 & 1.15 & 1.56 & 43.17 & + \\
\hline
\end{tabular}

(Chr) Known or estimated chromosome for the markers; $\left(M_{1}, M_{2}\right)$ markers flanking the putative QTL; (Dist) estimated distance ( $c M$ ) between $M_{1}$ and $M_{2}$; (QTL-Dist) estimated distance between $M_{1}$ and the putative QTL; $(F$ stat) $F$ statistic produced from the composite interval mapping model for the QTL; (Lod) lod score associated with the locus nearest the listed putative QTL obtained from a standard univariate interval mapping analysis using MAPMAKER (the precise locations producing maximum lod scores from univariate interval mapping analysis differed somewhat from those obtained using the multiple regression procedure); (Add $R^{2}$, Dom $R^{2}$ ) percentage of NaSBP variance explained by additive and dominance effects, respectively, by the putative $Q T L ;\left(C u m R^{2}\right)$ cumulative percentage of NaSBP variation explained; (Effect) net NaSBP effect of the SHR allele at the putative QTL: + denotes increase in NaSBP; denotes decrease.

${ }^{a} \mathrm{~A}$ putative QTL whose composite interval mapping $F$ statistic did not achieve significance but whose inclusion greatly increased the explanatory power of the model and was also associated with a marker with large effects on NaSBP (see text, Table 1, and Fig. 1). 


\section{SCHORK EI AL.}

cated with the sample size at our disposal, as noted in Methods. Also reported in Table 3 are lod scores obtained from a standard univariate interval mapping analysis using the MAPMAKER program (Lander et al. 1987). The reported lod scores are associated with putative QTL locations closest to the QTL locations obtained in the SC interval mapping analysis because the locations of putative QTLs obtained within the univariate and SC interval mapping analyses differed slightly. Lander and Schork (1994) suggest that for an interval mapping analysis of an intercross population, univariate lod scores of 3.3 should be taken as indicative of the presence of a QTL for a one degree-of-freedom test. As can be determined from Table 3, four of the six loci determined from our procedure produced lod scores $>3.3$, which corroborates the significance of the QTLs detected.

\section{DISCUSSION}

This study makes clear the power modern molecular genetic and biometrical analysis technologies possess in gene mapping experiments involving model organisms. Blood pressure is considered a classical polygenic or multifactorial trait whose genetic basis will be difficult to unravel. Despite this, we were able to characterize six unique genetic loci that simultaneously influence NaSBP variation in a model organism often used in BP and hypertension research. These loci can be examined as potential candidate loci in other types of study. Although the actual role of these loci in salt and BP regulation, and cardiovascular physiology in general, will remain obscure until further studies have been done, our results do suggest some important research directions. For instance, by refining or saturating the $\mathrm{BN} \times$ SHR genome with more markers, future studies should be able to better isolate both the locations of previously identified QTLs and altogether new QTLs existing in regions poorly covered by the markers used in this study. In addition, by characterizing the location of QTLs influencing BP, congenic strains can be created for which the ultimate (Wagner et al. 1992) developmental and physiologic significance of relevant genes can be studied (Festing 1979; Pravenec et al. 1989; Wagner et al. 1992). Furthermore, the lack of apparent epistatic interactions involving the putative QTLs determined from the analysis is of tremendous interest and should spark many methodological and biological questions.
Two points about our study demand serious attention and criticism, however. First, the primary phenotype in our analysis was NaSBP. It is possible that the loci we have detected have unique effects on NaSBP, with different genes working to influence other types of BP. Our rationale for using NaSBP was physiological: By sufficiently challenging the rats with salt, we felt that we would be able to effectively "wash out" or diminish interindividual differences in dietary and metabolic parameters that mediate salt regulation that could confound detection of basal BP genes if not accommodated or accounted for properly. This physiological consideration should impact interpretation of our results. It would be of interest to compare and contrast genes influencing other types of BP, including BP "responsiveness" (i.e., change from baseline) genes to salt (or other) challenges. Second, we focused on a $\mathrm{BN} \times \mathrm{SHR}$ cross. It is likely that strain-specific alleles and genetic backgrounds associated with other types of crosses may result in the detection of other (or additional) loci than the ones we were able to detect. This fact may help explain why we did not detect linkages to other BP loci reported in the literature. In addition, it would be of interest to gauge the contribution of $\mathrm{X}$ and $\mathrm{Y}$ chromosome loci, as well as other factors, such as age and weight, on NaSBP. Our multivariate regression approach to locus mapping and characterization could easily accommodate this, and we are actively pursuing such analyses.

Ultimately, our study represents one of the most complete genetic dissections of NaSBP variation to date and offers new candidate loci for human BP regulation (if relevant homologies between the rat and human genomes can be found) and provides further evidence that implementation of modern biometrical genome scan technologies should allow rapid progress to be made in the genetic dissection of complex, multigenic traits.

\section{METHODS}

\section{The Nature of the Cross}

The study examined NaSBP variation in intercross $\left(\mathrm{F}_{2}\right)$ progeny from a cross between the $\mathrm{BN}$ normotensive rat strain and the SHR; $235 \mathrm{~F}_{2}$ rats were available for study. NaSBP variation was studied because of previous success with this trait (Jacob et al. 1991) and because salt balance plays a crucial role in blood pressure regulation. The rats were phenotyped 16 weeks after birth over a 14-day period 


\section{MULTIGENIC BASIS OF BLOOD PRESSURE VARIATION}

to minimize potential seasonal variation in BP. The majority of the animals were studied within 12 weeks of each other. Baseline levels for SBP, diastolic blood pressure (DBP), mean arterial blood pressure (MAP), and heart rate (HR) were measured directly through an indwelling catheter in the femoral artery (Soato and Kreiger 1974; Buckingham 1976; Andrews et al. 1978). Immediately after establishing baseline parameters, the rats were given a diet that included water with $1.0 \% \mathrm{NaCl}$ for 13 days. At the end of 13 days, each rat was anesthetized and a catheter was placed in its contralateral femoral artery. SBP, DBP, MAP, and $\mathrm{HR}$ were remeasured on the day after this catheter was inserted. Of the 235 rats, 222 completed this phenotyping protocol. Of the 222 rats completing the protocol, 188 were then genotyped for 179 genetic markers following a standard protocol (Jacob et al. 1991). The 179 markers typed had excellent coverage of 19 rat autosomes and the $X$ chromosome (Table 4 ) and have been presented in detail elsewhere (Jacob et al. 1995).

\section{Statistical Methods}

QTLs influencing NaSBP variation exhibited by the $188 \mathrm{~F}_{2}$ rats used in this study were assessed through combinations and modifications of ANOVA techniques, regression tech-

\begin{tabular}{ccc} 
Table 4. & Summary of linkage map data \\
\hline Chr & Markers & Map length (cM) \\
\hline 1 & 10 & 109.4 \\
2 & 13 & 93.8 \\
3 & 9 & 71.1 \\
4 & 10 & 55.9 \\
5 & 15 & 79.4 \\
6 & 8 & 126.2 \\
7 & 10 & 97.1 \\
8 & 6 & 50.4 \\
9 & 4 & 59.9 \\
10 & 7 & 57.7 \\
11 & 5 & 16.5 \\
12 & 5 & 55.8 \\
13 & 7 & 70.2 \\
14 & 8 & 69.0 \\
15 & 5 & 50.0 \\
16 & 5 & 42.0 \\
17 & 5 & 42.0 \\
18 & 9 & 21.6 \\
19 & 4 & 70.9 \\
X & 5 & \\
a & 29 & \\
& & \\
\hline
\end{tabular}

(Chr) Chromosome; (Markers) number of markers assigned to a specific chromosome; (Map length) total estimated chromosomal coverage of the markers estimated by maximum likelihood multilocus linkage analysis using MAPMAKER assuming the Kosambi map function with error detection turned on.

aMarkers not currently assigned, statistically or biologically, to a particular linkage group or chromosome. niques, and the interval mapping procedures described by Lander and Botstein (1986, 1989), Haley and Knott (1992), and Zeng $(1993,1994)$. As emphasized, the goal of the analysis was to simultaneously assess and characterize multiple locus influences on NaSBP variation. The analytic strategy used to determine NaSBP-influencing QTLs was broken down into four steps or stages. Briefly, the first stage involved both the investigation of associations between genotypes observed at each of the 179 marker loci and NaSBP values and the creation of simple preliminary genetic models for the effects of possible QTLs residing near each marker. The second stage involved the simultaneous assessment of the impact of marker/QTL effects on NaSBP values large enough to warrant further consideration based on liberal statistical criteria applied in the first stage. The third stage involved refining the location and effect of each putative QTL passing more stringent statistical criteria applied in the second stage. The final analysis stage examined the ultimate significance of each QTL effect when considered together with the other QTL effects.

For the first stage, simple associations between the intercross rat progeny genotypes at each marker locus and NaSBP values were assessed using ANOVA techniques, with genotype information taken as an independent variable. Because $F_{2}$ progeny were used, we could determine which rats were homozygous for the $\mathrm{BN}$ allele (BB rats), which were heterozygous (BS rats), and which were homozygous for the SHR allele (SS rats). Both parametric and nonparametric (i.e., Kuskall-Wallis) ANOVA techniques were used (Neter et al. 1985). In addition, a linear regression-based genetic model (i.e., dominant, recessive, or additive/codominant) characterizing the effects of alleles at a putative QTLs near each marker was also determined by assigning simple indicator variables to the rats based on genotype information. This linear model was of the form

$$
y_{i}=b_{0}+b_{1} x_{i}+e_{i}
$$

where $y_{i}$ is the NaSBP value for the $i$ th rat $(i=1 \ldots N), b_{0}$ is an intercept term, $b_{1}$ is a regression coefficient associated with the marker considered in the model, and $e_{i}$ is an error or residual term that characterizes variation not explained by the marker locus-QTL effect. The $x_{i}$ terms were associated with the genotype information from the ith rat for the markers tested. It was assumed that SHR alleles at marker loci could influence (i.e., potentially raise) NaSBP in a dominant, codominant (additive), or recessive fashion. For a model of dominance, $x_{i}$ took on a value of 0 if the $i$ th rat's genotype was $\mathrm{BN}$ homozygous and 1 if heterozygous or SHR homozygous; for a model of codominance, $x_{i}$ took on a value of 0 for BN homozygotes, $1 / 2$ for heterozygotes, and 1 for SHR homozygotes; and for a recessive model, $x_{i}$ took on a value of 0 for $\mathrm{BN}$ homozygotes and heterozygotes and 1 for SHR homozygotes. The bestfitting genetic model of the three for each marker was determined as the model that produced the smallest least square error (Neter et al. 1985) and was taken to characterize the allelic effects of putative QTLs near the marker loci. Only marker loci producing ANOVA and regression model $F$-test statistic $P$ values $<0.01$ were considered significant in this analysis stage. Although it could be argued that a more stringent $P$-value could have been used given that the number of marker loci investigated was large, this stage of the analysis was considered to be a true screening or initial analysis stage and it is clear that a loss of linkage 


\section{SCHORK ET AL.}

information can occur when analyzing trait-influencing loci independently in univariate settings when multiple trait-influencing loci exist (Schork et al. 1993; Knapp et al. 1994; Zeng 1994).

The second stage of the analysis involved the simultaneous consideration of the marker effects producing significant results at the first stage. This simultaneous assessment was performed by using a stepwise multiple regression analysis. Predictor variables in this analysis were taken as the indicator variables assigned to the rats based on marker genotypes and the best-fitting genetic regression model associated with the marker loci as analyzed in the first stage. The basic model used in the stepwise multiple regression was of the form

$$
y_{i}=b_{0}+\sum_{j=1}^{M} b_{j} x_{j i}+e_{i}
$$

where $b_{0}$ is an intercept term, $b_{i}$ is a coefficient associated with the $j$ th marker in the model, $e_{i}$ is a residual term, and where the $x_{j i}$ terms encompass indicator variable information associated with the $j$ th marker in the model from the $i$ th rat. The stepwise regression procedure implemented in the MIDAS computer package was used to carry out the analysis (Guire and Fox 1976). Entry and exit criteria for the stepwise procedure were set at F-statistic $P$ values of 0.05 and 0.10 , respectively (Neter et al. 1985). Note that the number of markers, $M$, entering into the final model gives an estimate of the number of QTLs influencing NaSBP variation, but is limited in the sense that the genomic coverage of the markers used in the analysis was not complete (i.e., there may be a QTL located at a chromosomal location not covered by markers used in the analysis).

The third stage of the analysis involved the refinement of the approximate position and ultimate effect of possible multiple NaSBP QTLs through interval mapping methods. To this end, the extended linear model described by Zeng as a SC interval mapping model was used (Zeng 1994). The SC model allows the assessment of the contribution of a putative QTL to the trait of interest while fixing other QTL effects in the model (the QTLs fixed in the model are collectively referred to as "background effects") and is similar to a feature incorporated into the MAPMAKER computer program (Lander et al. 1987). Using the SC model, a search for additional QTL effects was made in addition to refining the position of the QTL effects associated with the marker loci found to be possibly linked to QTLs from stages 1 and 2 . This refinement was carried out by fixing the marker locus effects significant in stage 2 of the analysis in a linear model and then testing locations within genomic intervals defined by 179 markers used in the study (Lander and Botstein 1989; Zeng 1993, 1994). The model assumed in the stage 3 analysis was of the form

$$
y_{i}=b_{0}+b^{t, a} x_{i}^{t, a} \cdot x_{i}^{t, a}+b^{t, d} x_{i}^{t, d}+\sum_{j=1}^{M} b_{j}^{f} x_{j i}^{f}+e_{i}
$$

where $b_{0}$ and $e_{i}$, are defined in Equation 1 . The $b^{f}$ and $x_{j i}^{f}$ terms have the same interpretation as the $b_{j}$ and $x_{j i}$ terms used in the basic linear model used in the multiple regression procedure used in stage 2 of the analysis. They are used to characterize fixed or background QTL effects. The $x^{t, a}$ and $x^{t, d}$ terms are associated with the expected additive and dominance effects, respectively, of a putative test QTL and are computed for the $i$ th individual from genotypic information obtained from marker loci flanking the position of the putative QTL as described by Haley and Knott (1992). The $b^{t, a}$ and $b^{t, d}$ terms are regression parameters associated with the additive and dominance effects of the putative test QTL. Using this model, the $x^{t, a}$ and $x^{t, d}$ terms associated with each location flanked by marker loci were tested for QTL effects while controlling for the effects of previously identified QTLs (i.e., $x_{j i}^{f}$ terms) (Zeng 1993). Twenty-five uniformly spaced points within each interval were tested by deriving relevant $x^{t, a}$ and $x^{t, d}$ information. Inclusion of background or fixed QTL effects in an interval analysis model in this manner increases the power to resolve the location of putative QTLs because statistical noise is reduced (Zeng 1994). In addition, multilocus influences can be accounted for and assessed within this approach (Zeng 1994). We used the marker effects outlined in Table 2 as initial fixed QTL factors in equation 2; thus, $M$ was set equal to 5 . Because we used a select subset of the total marker loci in our marker pool, our model is consistent with the powerful SC interval mapping model proposed by Zeng (1994). The significance of putative QTLs from the SC interval mapping analysis was assessed through $F$ tests measuring the impact of a putative QTL on NaSBP variation. Adjustments to the $P$ values of the $F$-statistic significance tests were made to account for multiple comparisons using the strategy outlined by Zeng (1994). This strategy utilizes the number of intervals tested to correct for multiple comparisons. Because there were 131 intervals associated with the markers assigned to the 19 autosomes we investigated (i.e., the sum of the markers on the 19 autosomes less 19), we assumed an F-test critical value that had an associated $P$ value $0.05 / 131=0.0004$. Because both additive and dominance effects were considered for each putative QTL, appropriate $F$ tests had 2 degrees of freedom. For large samples, the appropriate $F$-test critical value is -7.75 .

When QTL effects were tested at a locus linked to a marker locus included in the background QTL effect set, that marker was dropped from the background set. In this way, we could refine the location of the QTLs associated with the markers initially fixed or included in the background set. No marker or QTL effect was eliminated from the background set for subsequent analyses, however, irrespective of the results of its re-analysis in the presence of the remaining background loci.

The final analysis stage involved computing expected additive and dominance factors for each putative QTL from relevant flanking marker and estimated location information using the Haley and Knott procedure (1992). These factors were then analyzed in a final multiple regression model (Neter et al. 1985) to assess the ultimate contribution of each putative QTL to NaSBP variation. Interactions among the putative loci also were assessed by multiplying appropriate terms. Although not an ideal way to investigate interactions, as we may have missed the effect of loci entering into interactions in the first stages of our analysis by virtue of their small effects when analyzed in isolation of the other locus they interact with, this assessment does allow us to gauge gross interactive effects among the loci with largest individual effects. 


\section{ACKNOWLEDGMENTS}

Computational work by N.J.S. was funded in part by grant HL48957-01 from The National Institutes of HealthNational Heart, Lung, and Blood Institute (NIH-NHLBI). J.E.K. is funded in part by grants from the Fundacao Amparo Pesquisa Estado Sao Paulo (91/0545-6), Agencia Finandora Projeptos-Brazil (66.93.0023.00), and Fundacao EJ Zerbini e Pro-Sangue-SP Brazil. Genotyping by H.J.J. was funded in part by grants from Bristol Myers-Squibb and grant DK47205-01 from the NIH. M.R.T. is funded by the American Heart Association, Florida affiliate (no. 9405003). Y.J.D. is funded in part by NIH grants HL-46631, HL-35610, and HL-35252. We thank Michael Boehnke, Aravinda Chakravarti, Alan Cowley, David Fulker, Andrew Green, Audrey Lynn, Richard Roman, Peter Tonellato, Alan Weder, and two anonymous reviewers for reading and commenting on earlier versions of this paper. E.L.S. is supported in part by NIH grant P50-HG00098-06.

The publication costs of this article were defrayed in part by payment of page charges. This article must therefore be hereby marked "advertisement" in accordance with 18 USC section 1734 solely to indicate this fact.

\section{REFERENCES}

Andersson, L., C.S. Haley, H. Ellegren, S.A. Knott, M. Johansson, K. Andersson, L. Andersson-Eklund, I. Edfors-Lilja, M. Fredholm, I. Hansson, J. Hakansson, and K. Lundstrom. 1994. Genetic mapping of quantitative trait loci for growth and fatness in pigs. Science 263: $1771-1774$.

Andrews, D.I., D.R. Jones, and F.O. Simpson. 1978. Direct recording of arterial blood pressure and heart rate in the conscious rat. J. Pharm. Pharmacol. 30: 524-525.

Buckingham, R.E. 1976. Indwelling catheters for direct recording of arterial blood pressure and intravenous injection of drugs in the conscious rat. J. Pharm. Pharmacol. 28: 459-461.

Davies, J.L., Y. Kawaguchi, S.T. Bennett, J.B. Copeman, H.J. Cordell, L.E. Pritchard, P.W. Reed, S.C.L. Gough, S.C. Jenkins, S.M. Palmer, K.M. Balfour, B.R. Rowe, M. Farrall, A.H. Barnett, S.C. Bain, and J.A. Todd. 1994. A genome-wide search for human type 1 diabetes susceptibility genes. Nature 371: 130-136.

Deng, A.Y., H. Dene, M. Pravenec, and J.P. Rapp. 1994a. Genetic mapping of two new blood pressure quantitive trait loci in the rat by genotyping endotyping endothelin system genes. J. Clin. Invest. 93: 2701-2709.

Deng, A.Y., H. Dene, and J.P. Rapp. 1994b. Mapping of a quantitative trait locus for blood pressure on rat chromosome 2. J. Clin. Invest. 94: 431-436.

Dubay, C., M. Vincent, N.J. Samani, P. Hilbert, M.A. Kaiser, J.P. Beressi, J. Kotelevtsev, J.S. Beckmann, F. Soubrier, J. Sassard, and G.M. Lathrop. 1993. Genetic determinants of diastolic and pulse pressure map to different loci Lyon hypertensive rats. Nature Genet. 3: 354-357.

\section{MULTIGENIC BASIS OF BLOOD PRESSURE VARIATION}

Festing, M.F.W. 1979. Inbred strains in biomedical research. Oxford University Press, New York, NY.

Guire, K.E. and D.J. Fox. 1976. Documentation for MIDAS. University of Michigan Statistical Research Laboratory, Ann Arbor, MI.

Haley, C.S. and S.A. Knott. 1992. A simple regression method for mapping quantitative trait loci in line crosses using flanking markers. Heredity 69: 315-324.

Hamet, P., D. Kong, M. Pravenec, J. Kunes, V. Kren, P. Klir, Y.L. Sun, and J. Tremblay. 1992. Restriction fragment length polymorphism of hsp70 gene, localized in the RT1 complex, is associated with hypertension in spontaneously hypertensive rats. Hypertension 19: $611-614$.

Harris, E.L., H. Dene, and J.P. Rapp. 1993. SA gene and blood pressure cosegregation using Dahl salt-sensitive rats. Am. J. Hypertension 6: 330-334.

Hashimoto, L., C. Habita, J.P. Beressi, M. Delepine, C. Besse, A. Cambon-Thomsen, I. Deschamps, J.I. Rotter, S. Djouiah, M.R. James, P. Froguel, J. Weissenbach, G.M. Lathrop, and C. Julier. 1994. Genetic mapping of a susceptibility locus for insulin dependent diabetes mellitus on chromosome 11q. Nature 371: 161-164.

Hilbert, P., K. Lindpaintner, J.S. Beckmann, T. Serikawa, F. Soubrier, C. Dubay, P. Catwright, B. De Gouyon, C. Julier, S. Takahasi, M. Vincent, D. Ganten, M. Georges, and G.M. Lathrop. 1991. Chromosomal mapping of two genetic loci associated with blood-pressure regulation in hereditary hypertensive rats. Nature 353: 521-529.

Jacob, H.L., K. Lindpaintner, S.E. Lincoln, K. Kusumi, R.K. Bunker, Y.-P. Mao, D. Gaqnten, V.J. Dzau, and E.S. Lander. 1991. Genetic mapping of a gene causing hypertension in the stroke-prone spontaneously hypertensive rat. Cell 67: 213-224.

Jacob, H.J., D.M. Brown, R.K. Bunker, M.J. Daly, V.J. Dzau, A. Goodman, G. Koike, V. Kren, T. Kurtz, A. Lernmark, G. Levan, Y.-P. Mao, A. Pettersson, M. Pravenec, J.S. Simon, C. Szpirer, J. Szpirer, M.R. Trolliet, E.S. Winer, and E.S. Lander. 1995. A genetic linkage map of the laboratory rat, Rattus norvegicus. Nature Genet. 9: 63-69.

Knapp, M., S.A. Seuchter, and M.P. Baur. 1994. Two-locus disease models with two marker loci: The power of affected-sib-pair tests. Am. J. Hum. Genet. 55: $1030-1041$.

Lander, E.S. and D. Botstein. 1986. Mapping complex genetic traits in humans: New strategies using a complete RFLP linkage map. Cold Spring Harbor Symp. Quant. Biol. 51: 46-61.

1989. Mapping Mendelian factors underlying quantitative traits using RFLP linkage maps. Genetics 121: 185-199. 


\section{SCHORK ET AL.}

Lander, E.S. and N.J. Schork. 1994. Genetic dissection of complex traits. Science 265: 2037-2048.

Lander, E.S., P. Green, J. Abrahamson, A. Barlow, M.J. Daly, S.E. Lincoln, and L. Newburg. 1987. MAPMAKER: An interactive computer package for constructing primary genetic linkage maps of experimental and natural populations. Genomics 1: 174-181.

Lindpaintner, K., P. Hilbert, D. Ganten, B. Nadal-Ginard, T. Inagami, and N. Iwai. 1993. Molecular genetics of the SA-gene: Cosegregation with hypertension and mapping to rat chromosome 1. J. Hypertension 11: 19-23.

Neter, J., W. Wasserman, and M.H. Kutner. 1985. Applied linear statistical models. Richard D. Irwin, Homewood, IL.

Pravenec, M., P. Klir, V. Kren, J. Zicha, and J. Kunes. 1989. An analysis of spontaneous hypertension in spontaneously hypertensive rats by means of new recombinant inbred strains. J. Hypertension 7: 217-221.

Pravenec, M., V. Kren, J. Kunes, A.G. Scicli, O.A. Carretero, L. Simonet, and T.W. Kurtz. 1991. Cosegregation of blood pressure with a kallikrein gene family polymorphism. Hypertension 17: 242-246.

Rapp, J.P., S.M. Wang, and H. Dene. 1989. A genetic polymorphism in the renin gene of Dahl rats cosegregates with blood pressure. Science 243: 542-544.

Rapp, J.P., H. Dene, and A.Y. Deng. 1994. Seven renin alleles in rats and their effects on blood pressure. $J$. Hypertension 12: 349-355.

Risch, N., S. Ghosh, and J.A. Todd. 1993. Statistical evaluation of multiple-locus linkage data in experimental species and its relevance to human studies: Application to nonobese diabetic (NOD) mouse and human insulin-dependent diabetes mellitus (IDDM). Am. J. Hum. Genet. 53: 702-714.

Samani, N.J., D. Lodwick, M. Vincent, C. Dubay, M.A. Kaiser, M.P. Kelly, M. Lo, J. Harris, J. Sassard, M. Lathrop, and J.D. Swales. 1993. A gene differentially expressed in the kidney of the spontaneously hypertensive rat cosegregates with increased blood pressure. J. Clin. Invest. 92: 1099-1103.

Schork, N.J., M. Boehnke, J.T. Terwilliger, and J. Ott. 1993. Two-trait-locus linkage analysis: A powerful strategy for mapping complex traits. Am. J. Hum. Genet. 53: $1127-1136$.

Soato, G.G. and E.M. Kreiger. 1974. Heart rate after acute hypertension in the rat. Am. J. Physiol. 227: 1389-1393.

Tanksley, S.D. 1993. Mapping polygenes. Annu. Rev. Genet. 27: 205-233.

Wagner, J., K. Zeh, and M. Paul. 1992. Transgenic rats in hypertension research. J. Hypertension 10: 601-605.

Ward, R. 1990. Familial aggregation and genetic epidemiology of blood pressure. In Hypertension: Pathophysiology, diagnosis, and management (ed. J.H. Laragh and B.M. Brenner), pp. 81-100. Raven Press, New York, NY.

Zeng, Z.-B. 1993. Theoretical basis of separation of multiple linked gene effects on mapping quantitative trait loci. Proc. Nat. Acad. Sci. 90: 10972-10976.

. 1994. Precision mapping of quantitative trait loci. Genetics 136: 1457-1468.

Received June 15, 1995; accepted in revised form August 9, 1995. 


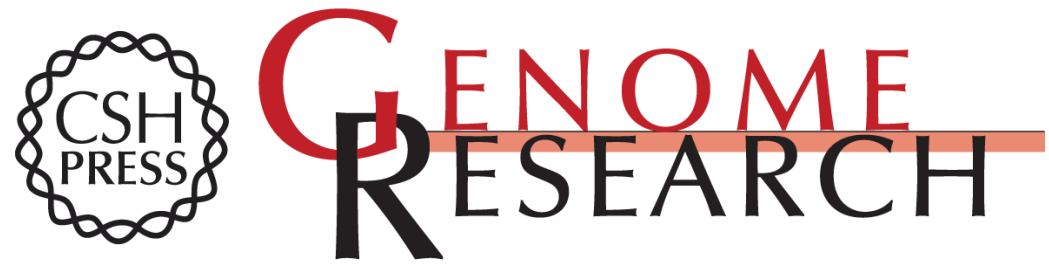

\section{A biometrical genome search in rats reveals the multigenic basis of blood pressure variation.}

N J Schork, J E Krieger, M R Trolliet, et al.

Genome Res. 1995 5: 164-172

Access the most recent version at doi:10.1101/gr.5.2.164

References This article cites 32 articles, 7 of which can be accessed free at:

http://genome.cshlp.org/content/5/2/164.full.html\#ref-list-1

\section{License}

Email Alerting Receive free email alerts when new articles cite this article - sign up in the box at the Service top right corner of the article or click here.

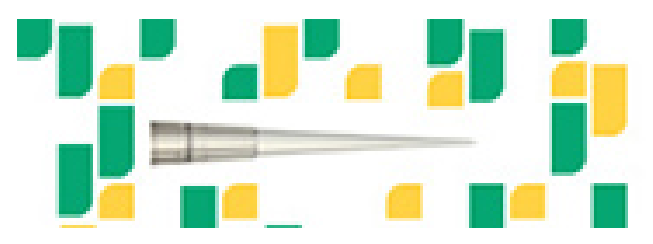

To subscribe to Genome Research go to: https://genome.cshlp.org/subscriptions 